\title{
Exposición selectiva y sus efectos en el comportamiento electoral de los ciudadanos: la influencia del consumo mediático en el voto en las elecciones generales españolas de 2015 y 2016
}

\section{José María Ramírez-Dueñas ${ }^{1}$ María Lourdes Vinuesa-Tejero²}

Recibido: 14/03/2019

Aprobado por pares: 08/05/2019
Enviado a pares: $16 / 03 / 2019$

Aceptado: 15/08/2019

DOI: $10.5294 /$ pacla.2020.23.4.6

Para citar este artículo / to reference this article / para citar este artigo

Ramírez-Dueñas, J. M. y Vinuesa-Tejero, M. L. (2020). Exposición selectiva y sus efectos en el comportamiento electoral de los ciudadanos: la influencia del consumo mediático en el voto en las elecciones generales españolas de 2015 y 2016. Palabra Clave, 23(4), e2346. https://doi.org/10.5294/pacla.2020.23.4.6

\section{Resumen}

La nueva tendencia de polarización política de los medios de comunicación en España lleva a los autores de este trabajo a replantearse la relación entre medios de comunicación y ciudadanos dentro de la teoría de la exposición, percepción y retención selectiva y su influencia en el comportamiento electoral. La selección de contenidos de información política que llevan a cabo los ciudadanos durante las campañas electorales conforma una dieta que afecta a su voto. Por ello, en este artículo de investigación, se intenta esclarecer la relación existente entre el consumo de información política a través de diferentes medios de comunicación de masas y el comportamiento electoral de los votantes desde la nueva realidad mediática existente en el país. Para ello, y según las encuestas poselectorales del Cen- 
tro de Investigaciones Sociológicas (CIS), se han estudiado los efectos en el voto de la exposición mediática de los ciudadanos en las elecciones generales de 2015 y las de 2016, aquellas en las cuales se produjo una crisis en el sistema de partidos en España tras varias décadas de bipartidismo y estabilidad institucional. Los resultados permiten concluir que la discusión política en el país se encuentra estructurada en dos ejes de confrontación: el ideológico (izquierda-derecha), pero también uno territorial, que polariza políticamente aquellos ciudadanos de territorios con identidad territorial propio (principalmente Cataluña). De esta forma, la narrativa política está altamente influenciada por los medios, ya que los electores utilizan el consumo mediático como símbolo de reafirmación electoral siguiendo la información política de medios afines.

\section{Palabras clave (Fuente: tesauro de la Unesco)}

Medios de comunicación de masas; elecciones; España; comunicación política; comportamiento político; prensa; radio; televisión 


\section{Selective Exposure and its Effects on Citizens' Electoral Behavior: The Influence of Media Consumption on Voting in the Spanish General Elections of 2015 and 2016}

\section{Abstract}

The new trend of politically polarized media in Spain has led the authors of this paper to rethink the relationship between media and citizens within selective exposure, perception and retention theory and its influence on electoral behavior. The selection of political information contents by citizens during electoral campaigns forms a diet that influences their votes. Therefore, this research article attempts to clarify the relationship between the consumption of political information from mass media and the voters' electoral behavior amid the new media reality of the country. For this, and per the post-electoral surveys of the Center for Sociological Research (CIS, for its acronym in Spanish), it studies the effects of citizens' media exposure during the 2015 and 2016 general elections on voting. In these years, there was a crisis in the party system in Spain after several decades of bipartisanship and institutional stability. The results allow concluding that the political discussion in the country builds on two lines of confrontation: ideological (left-right) and territorial, which politically polarizes those citizens from territories with their own identity (mainly Catalonia). Media positively influence the political narrative since voters consume political information from related media as a symbol of electoral reaffirmation.

\section{Keywords (Source: Unesco Thesaurus)}

Mass media; elections; Spain; political communication; political behaviour; press; radio; television 


\section{Exposição seletiva e seus efeitos no comportamento eleitoral dos cidadãos: a influência do consumo midiático no voto nas eleições gerais espanholas de 2015 e 2016}

\section{Resumo}

A nova tendência de polarização política dos meios de comunicação na Espanha leva os autores deste trabalho a repensar a relação entre meios de comunicação e cidadãos dentro da teoria da exposição, percepção e retenção seletiva, e sua influência no comportamento eleitoral. A seleção de conteúdos de informação política que os cidadãos realizam durante as campanhas eleitorais conforma uma dieta que afeta seu voto. Por isso, neste artigo de pesquisa, pretende-se esclarecer a relação existente entre o consumo de informação política por meio de diferentes meios de comunicação de massas e o comportamento eleitoral dos votantes desde a nova realidade midiática existente no país. Para isso e segundo as pesquisas pós-eleitorais do Centro de Pesquisas Sociológicas, estudam-se os efeitos no voto da exposição midiática dos cidadãos nas eleições gerais de 2015 e das de 2016, aquelas nas quais uma crise é produzida no sistema de partidos na Espanha após várias décadas de bipartidismo e estabilidade institucional. Os resultados permitem concluir que a discussão política no país se encontra estruturada em dois eixos de enfrentamento: o ideológico (esquerda-direita), mas também um territorial, que polariza politicamente aqueles cidadãos de territórios com identidade territorial próprio (principalmente Catalunha). Dessa forma, a narrativa política está altamente influenciada pelos meios, já que os eleitores utilizam o consumo midiático como símbolo de reafirmação eleitoral seguindo a informação política de meios afins.

\section{Palavras-chave (Fonte: tesauro da Unesco)}

Meios de comunicação de massa; eleições; Espanha; comunicação política; comportamento político; pressione; rádio; televisão 


\section{Introducción}

El sistema mediático español, catalogado como pluralista polarizado según la clasificación de Hallin \& Mancini (2004), se ha visto reforzado en la actualidad con el descenso del consumo de la prensa impresa, la instrumentalización de los medios de comunicación al servicio del poder o el aumento de su polarización partidista, así como el papel de los medios online, asignatura pendiente de las investigaciones.

Este escenario de polarización es lo que hemos considerado como propicio para la realización de esta investigación, con el fin de que nos permitiera dar respuesta a la relación existente entre el consumo mediático de los ciudadanos y su comportamiento electoral. Para ello, hemos pretendido seguir con la línea de algunas de las investigaciones sobre los efectos políticos de los medios de comunicación en el ámbito anglosajón y en España. Esa relación entre medios y votantes, dentro del proceso de la comunicación política, ha sido explicada desde distintas perspectivas, en función del papel que se adjudicase a los medios y al sistema mediático. Con el aumento de medios de comunicación y, por tanto, con el incremento del volumen de información que reciben los ciudadanos, en nuestro caso, información política, los votantes tienen una mayor capacidad para seleccionar contenidos según sus parámetros de afinidad.

Lo anterior ha determinado que la perspectiva de análisis de la que partimos en esta investigación sea la teoría de la exposición selectiva que plantearon en la década de los cuarenta Lazarsfeld et al. (1944).

\section{La teoría de la exposición selectiva}

Lazarsfeld et al. (1944) enunciaban la influencia de los medios de comunicación y la proactividad de los ciudadanos a la exposición a aquellos mensajes y canales/cabeceras de prensa más afines o cercanos a sus opiniones y a rehuir aquellos que le son hostiles o disonantes.

En ese sentido, Festinger (1957), en su teoría de la disonancia cognitiva, pretendió dar respuesta a este comportamiento de los individuos de evitar la información incoherente/inconsistente con sus opiniones pre- 
existentes como una forma de reducir su disonancia intraindividual. La investigación de este patrón psicológico desde el punto de vista mediático y político (conocido como partisan selective exposure en la literatura) ha sido especialmente estudiada a partir de la década de los noventa, aun existiendo investigaciones en los años previos. Por ejemplo, Sears \& Freedman (1967) intentaron ahondar en el papel activo de los ciudadanos en la selectividad de la información. Chaffee \& Miyo (1983) se centraron en el papel de refuerzo de exposición selectiva de los ciudadanos ante sus planteamientos ideológicos en las campañas electorales.

Pero es especialmente en los últimos veinte años en los que encontramos un mayor interés académico sobre consumo mediático y exposición selectiva partidista. Por un lado, buena parte de la investigación ha utilizado la ideología política como indicador para evaluar la afirmación o disonancia cognitiva en mensajes políticos en los medios de comunicación (Rinke \& Lück, 2010, pp. 228; Vraga, 2015). Por otro, algunos investigadores han puesto la relevancia en el papel activo y seleccionador de los individuos. La selectividad (selectivity) de la información elegida (ya enunciado por Sears \& Freedman, 1967) implica la necesidad de involucración del individuo sobre su consumo mediático: los participantes eligen medios de comunicación que prevén tener mayor sintonía con su afinidad política e ideológica (Iyengar \& Hahn, 2009), y pasan más tiempo leyendo información coherente que aquella que es disonante con sus opiniones (Graf \& Aday, 2008).

Los ciudadanos luchan por mantener su coherencia, evitan información que ataque a sus creencias o afiliaciones políticas y reducen la diversidad de opiniones y posturas que reciben. De esta forma, se ha producido un aumento del interés académico por la devaluación del concepto opinión pública y su carácter uniformador por intereses comunes de la sociedad (Ksiazek, 2016, pp. 216-217).

Los consumidores políticamente polarizados están motivados a ejercer una mayor selectividad en sus opciones de noticias. A medida que las audiencias se vuelven más polarizadas, especialmente en cuestiones de información política, los medios de comunicación participan con mayores 
sesgos en un intento de ganar cuota de mercado y satisfacer a su base de consumidores (Iyengar \& Hahn, 2009). La tendencia de los ciudadanos a buscar contenidos mediáticos más cercanos a sus posiciones fuerza la polarización política y, a su vez, de los medios de comunicación. La polarización y la exposición selectiva partidista están generando "un público menos tolerante y más fragmentando" (Bou-Hamad \& Yehya, 2016), que, al mismo tiempo, está dispuesto a consumir informaciones adversas a sus posiciones, porque le refuerzan aún más sus opiniones (Stroud, 2010, pp. 569-570). Los medios de comunicación producen reforzamientos de las predisposiciones de los individuos a medida que las audiencias se convierten en subconjuntos (Bennett \& Iyengar, 2008, p.720; Tsfati et al., 2014). Bajo este prisma, los medios han iniciado una tendencia de marcar aún más sus posiciones partidistas (y editoriales) para fidelizar a sus votantes.

Como consecuencia de la mayor predisposición a consumir contenidos coherentes con nuestras opiniones, los ciudadanos están más polarizados y menos informados (Levendusky, 2013; Prior, 2013; Stroud, 2010), especialmente cuando los medios de comunicación tratan temas controvertidos (Iyengar \& Hahn, 2009). Recientes informes del Pew Research Center (Mitchell et al., 2014) alertan de un empeoramiento de la cobertura de los temas, informaciones más sesgadas y desinformación.

En ese sentido, dos tendencias podrían marcar un aumento de la exposición selectiva y del papel activo de los individuos en relación con su consumo de información política. Respecto de los efectos de la exposición a través de redes sociales en el comportamiento electoral de los ciudadanos (Dimitrova et al., 2014; Gil de Zúñiga et al. 2017; Lee \& Kim, 2017; Messing \& Westwood, 2014), y pese a que Lee \& Xenos (2019, p. 23) exponen algunas de las limitaciones de la exposición selectiva, se está planteando que los usuarios no solo reciben información política y participan en redes sociales, sino que también están expuestos de manera incidental a ellas, incluso en contenidos que no son coherentes con su afiliación ideológica o partidista. Y, por otro lado, existe una incidencia del fenómeno de la fragmentación de audiencias fruto de los nuevos modelos de consumo audiovisual (nuevos canales on demand, medios digitales, etc.) en la selección de la información política (Fletcher \& Nielsen, 2017). 
Tanto España como en América Latina, las investigaciones sobre la intensidad de la exposición selectiva han certificado el impacto en los votantes de aquellos medios o informaciones más cercanos a sus opiniones personales y el impacto de la prensa digital y las redes sociales como mecanismos de reforzamiento de las posiciones políticas de los electores.

Más en concreto, el análisis de Martín-Llaguno \& Berganza-Conde (2001, p. 69) centrado en las elecciones generales de 1996 (con un doble método de análisis de contenido con encuesta estadística en Navarra) pretendió demostrar el alto índice de partidismo de los medios de comunicación en España y la relación existente entre el consumo mediático de los individuos hacia prensa y televisión —en este caso- afín a sus opiniones personales. Por su parte, Humanes (2014, p. 782), en su estudio sobre consumo de información política durante las campañas electorales de 2008 y 2011, expone que el periodismo español está altamente politizado y que responde al incremento de la polarización política y la alineación ideológica de los ciudadanos. En su análisis, Humanes demuestra que la prensa es el medio con mayor intensidad de exposición selectiva, mientras que la televisión, dada sus mayores audiencias y su más amplio perfil comercial, no ha asumido con tanta intensidad el valor de la polarización partidista. Sus resultados, además, advierten que los individuos cambian su fuente de información cuando detectan que la línea editorial del medio de comunicación se ha modificado (p. 795). Finalmente, Fernández y Rodríguez-Virgili (2017) han constatado en España, Argentina y Venezuela un desplazamiento de los medios tradicionales de consumo de información política a prensa digital, y en el último lustro, hacia las redes sociales (Facebook, Twitter, WhatsApp y Telegram, especialmente), así como los sesgos informativos que ello supone.

\section{El impacto de los medios de comunicación en el voto}

Ahora bien, ¿qué efectos tiene esta exposición selectiva en el comportamiento electoral de los votantes? Anteriormente, diagnosticábamos la incidencia que tenían los medios de comunicación en su papel de refuerzo de las opiniones de los votantes y la incidencia de la afiliación partidista como movilizador de los sesgos de interpretación de los individuos. 
Sin embargo, los primeros estudios del comportamiento electoral minusvaloraron la influencia electoral de los medios de comunicación. La teoría sociología del voto (también llamada escuela de Columbia) puso el foco en los componentes sociodemográficos de los votantes para dictaminar el sentido del voto. Lipset \& Rokkan (1967) analizaron las alineaciones partidistas bajo el prisma de las fracturas sociales (cleavages), pero ignoraron el papel de los medios de comunicación más allá de advertir el papel que podrían tener en el futuro.

Hasta las investigaciones de la escuela de Míchigan, más conocida como teoría psicológica, no se tomó en consideración la importancia que podrían tener los medios de comunicación entre los factores de alineación partidista y electoral de los votantes. Al igual que la familia, la escuela o la cultura nacional, los media deben ser considerados como variables de "socialización" (Anduiza \& Bosch, 2012, pp. 190-196), en que el votante adquiere elementos de juicio para dirimir entre opciones electorales.

Los ciudadanos, que habían adquirido a través de esas fuentes de socialización los valores y las posiciones políticas, serían más proclives a votar a un partido u otro en función de sus valores. La teoría de exposición selectiva trasladaría este factor a su modelo de acceso de información, y más sabiendo el papel central de las campañas electorales (y el interés por ellos de los medios de comunicación) que demostraron las investigaciones de Campbell et al. (1960, p. 543).

En ese sentido, la discusión académica se centra entre aquellos que defienden lo que se ha conocido como "efectos mínimos" (Lazarsfeld et al., 1944), es decir, aluden al papel secundario de los medios de comunicación consecuencia de la activación, la conversión y el refuerzo frente a la capacidad de influencia de otros miembros del grupo donde el individuo esté presente. Años más tarde, Katz \& Lazarsfeld (1955) profundizarán en el impacto de la influencia personal y allí desarrollarán la hipotesis de la influencia de los mensajes políticos en función de la voluntad del individuo de exponerse a unos u otros contenidos.

Es esta línea de los efectos mínimos de los medios de comunicación en que se han centrado los investigadores que han pretendido estudiar los 
efectos de la exposición selectiva en el voto: activación, conversión y refuerzo, de modo que es este último el principal foco de atención debido a la polarización partidista cada vez más fuerte de la prensa (Bennett \& Iyengar, 2008; Dilliplane, 2014; Hernández-Santaolalla, 2018).

\section{El consumo de medios en las campañas electorales de 2015 y 2016 en España}

Como ya hemos comentado, el sistema mediático español se caracteriza por el incremento de canales analógicos y la proliferación de digitales. Además, se trata de un sistema pluralista polarizado (Hallin \& Mancini, 2004) en el que los medios se alejan de la premisa periodística de servicio público para convertirse en instrumentos partidistas en torno a la existencia de dos polos ideológicos (izquierda-derecha), tal y como recogemos en la figura 1.

\section{Figura 1. Ubicación ideológica percibida de los medios en España.}

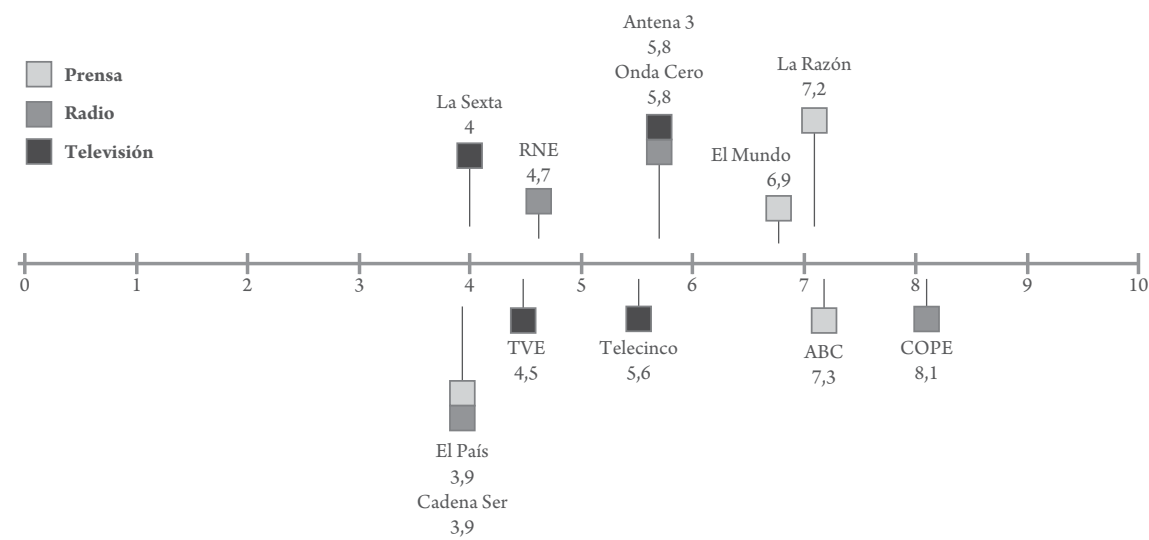

Fuente: Humanes (2014, p. 788) y Farias (2010).

Este sistema está configurado por tres variables:

- La estratificación de los canales en función de variables sociodemográficas.

- La unidireccionalidad del consumo televisivo, de modo que los individuos que se informan por prensa o radio suelen hacerlo también por televisión. 
- Las diferencias existentes entre consumo de canales, con el dominio de la televisión, y en menor medida, de la prensa y de la radio.

El consumo mediático de la televisión como medio más importante para la información política en campañas electorales sigue siendo dominante. Tanto en las elecciones de diciembre de 2015 como en las de junio de 2016, observamos que más de la mitad de los electores se informó diariamente por la televisión, mientras que aquellos que lo hicieron por la radio o la prensa se situaron en torno al $20 \%$. Al contrario, solo uno de cada cinco electores no utilizó la televisión como medio de información política, frente al $60 \%$, de media, que no usaron ni radio ni prensa. En líneas generales, en torno a un $85 \%$ de la población se informó por televisión, un $40 \%$ por la prensa y algo más del $30 \%$ por radio (tabla 1 ).

Tabla 1. Consumo mediático de información política

\begin{tabular}{|l|c|c|c|c|c|c|}
\hline \multirow{2}{*}{} & \multicolumn{2}{|c|}{ Prensa } & \multicolumn{2}{c|}{ Radio } & \multicolumn{2}{c|}{ Televisión } \\
\cline { 2 - 7 } & $\mathbf{2 0 1 5}(\%)$ & $\mathbf{2 0 1 6}(\%)$ & $\mathbf{2 0 1 5}(\%)$ & $\mathbf{2 0 1 6}(\%)$ & $\mathbf{2 0 1 5}(\%)$ & $\mathbf{2 0 1 6}(\%)$ \\
\hline Todos o casi todos los días & 23 & 19,8 & 19,6 & 17,5 & 58,3 & 50,8 \\
\hline Cuatro o cinco días por semana & 4,4 & 4,7 & 3,4 & 3,7 & 10,1 & 11,3 \\
\hline Dos o tres días por semana & 6,1 & 5,4 & 3,9 & 2,8 & 7,7 & 7,2 \\
\hline Solo los fines de semana & 3,3 & 2,5 & 0,9 & 0,6 & 1,6 & 1,2 \\
\hline De vez en cuando & 8,5 & 7,2 & 7,5 & 5,9 & 9,2 & 8,1 \\
\hline Nunca o casi nunca & 54,2 & 59,9 & 63,7 & 68,8 & 12,9 & 21,1 \\
\hline N. C. & 0,5 & 0,5 & 1 & 0,6 & 0,3 & 0,3 \\
\hline Total & 100 & 100 & 100 & 100 & 100 & 100 \\
\hline
\end{tabular}

N. C.: no contesta.

Fuente: elaboración propia según encuestas poselectorales del Centro de Investigaciones Sociológicas (CIS) n.os 3126 y 3145.

Pero ese mayor consumo de la televisión no ha impedido que esta encabece también un mayor descenso del consumo de la información política, que, por otro lado, también se ha dado en el resto de medios. Este descenso se observa especialmente en la categoría de consumo diario, pero, además, un repunte de entre cinco (radio y prensa) y ocho puntos (televisión) de aquellos que no lo hicieron por ningún canal. Los ciudadanos tienden a desconectar en campañas electorales, y así como se produjo una dismi- 
nución de la participación electoral entre ambos comicios (de cuatro puntos), se reproduce del mismo modo en el consumo televisivo.

Pero, al margen de este dato, y en lo que respecta a nuestro estudio, hay que decir que en España el consumo de medios por parte de los votantes es elevado y las principales diferencias en cuanto a elección del canal, según las encuestas poselectorales del CIS de 2015 (n. $\left.{ }^{\circ} 3126\right)$ y 2016 (n. ${ }^{\circ} 3145$ ), vienen dadas por la procedencia ideológica o territorial.

Otro aspecto es que el consumo de información política se encuentra muy concentrado en el caso de la televisión y la radio, pero muy fragmentado en la prensa por la multitud de periódicos regionales y locales. La televisión se concentra fundamentalmente en La Sexta, Televisión Española (TVE1) y Antena 3 (aproximadamente un $20 \%$ ), con Telecinco (datos cercanos al $15 \%$ ) y Cuatro (5\%). Solo se rompe esta dinámica en Cataluña, con el dominio de la televisión pública catalana, TV3. Lo mismo sucede con la radio, en que el $70 \%$ de los oyentes se encuentra en Cadena Ser (casi un tercio del total), Cope, Onda Cero y Radio Nacional de España (RNE), sin prejuicio de que emisores regionales (especialmente RAC1 en Cataluña y Radio Euskadi) consigan cuotas de audiencias significativas en sus respectivas comunidades.

La prensa, en cambio, posee un carácter más atomizado, ya que entre los grandes cinco periódicos (El País, El Mundo, ABC, La Razón y Público) no superan el $40 \%$ del consumo de información política. Esta fragmentación viene por la prensa regional, especialmente en Cataluña, con La Vanguardia y El Periódico, y la local, que poseen una alta cuota de mercado en sus respectivas zonas.

\section{Metodología}

En esta investigación, con el fin de constatar la existencia de exposición selectiva, hemos utilizado el método cuantitativo de análisis mediante encuestas para analizar los efectos políticos de los medios de comunicación sobre el electorado español. Más en concreto, para observar la influencia de la exposición selectiva en el nuevo escenario multipardista, hemos realizado un análisis de las elecciones generales de diciembre de 2015 y de las eleccio- 
nes generales de junio de 2016, dos procesos electorales que vinieron marcados por la aparición de dos nuevos partidos (Podemos y Ciudadanos) y por la pérdida de la hegemonía del Partido Popular (PP) y del Partido Socialista Obrero Español (PSOE).

Este periodo interelectoral demostró no solo una mayor polarización existente en el eje ideológico (izquierda-derecha), sino también partidista dentro del nuevo sistema de partidos emergente (según afinidad mediática hacia las cuatro formaciones). Junto a ella, el problema sobre la independencia de Cataluña abrió nuevos ejes de discusión propios de las particularidades de la estructura autonómica de España (tensión centro-periferia).

Para la realización de este artículo de investigación, se han utilizado las encuestas poselectorales del CIS correspondientes a las convocatorias electorales de 2015, estudio de caso 1 (n. $\left.{ }^{\circ} 3126\right)$, y 2016, estudio de caso 2 (n. $\left.{ }^{\circ} 3145\right)$.

La primera se realizó del 7 de enero al 19 de marzo con 6242 entrevistas y la segunda del 2 al 21 de julio con 6175 entrevistas. Estos estudios son muy valiosos por aportar los datos sobre el consumo mediático de los españoles y la adscripción de su voto (tabla 2).

Tabla 2. Ficha técnica de las encuestas utilizadas

\begin{tabular}{|c|c|c|}
\hline & $\begin{array}{c}\text { Estudio de caso } 1 \\
\text { elecciones generales } 2015\end{array}$ & $\begin{array}{c}\text { Estudio de caso } 2 \\
\text { elecciones generales } 2016\end{array}$ \\
\hline Encuesta & N.o 3126 & N.o 3145 \\
\hline Ámbito & Nacional & Nacional \\
\hline Universo & $\begin{array}{c}\text { Población española de ambos sexos de } 18 \\
\text { años y más }\end{array}$ & $\begin{array}{c}\text { Población española de ambos sexos de } 18 \\
\text { años y más }\end{array}$ \\
\hline Tamaño de la muestra & $\begin{array}{l}\text { Diseñada: } 7798 \text { entrevistas } \\
\text { Realizada: } 6242 \text { entrevistas }\end{array}$ & $\begin{array}{l}\text { Diseñada: } 6250 \text { entrevistas } \\
\text { Realizada: } 6175 \text { entrevistas }\end{array}$ \\
\hline Puntos de muestreo & 694 municipios y 50 provincias & 524 municipios y 50 provincias \\
\hline Error muestral & $\begin{array}{l}\text { Para un nivel de confianza del } 95,5 \% \text { (dos } \\
\text { sigmas), y } \mathrm{P}=\mathrm{Q} \text {, el error es de } \pm 1,3 \% \text { para el } \\
\text { conjunto de la muestra, y en el supuesto de } \\
\text { muestreo aleatorio simple }\end{array}$ & $\begin{array}{l}\text { Para un nivel de confianza del 95,5\% (dos } \\
\text { sigmas), y P = Q el error es de } \pm 1,27 \% \text { para } \\
\text { el conjunto de la muestra, y en el supuesto de } \\
\text { muestreo aleatorio simple }\end{array}$ \\
\hline Fecha de realización & Del 7 de enero al 19 de marzo de 2016 & Del 2 al 21 de julio de 2016 \\
\hline
\end{tabular}

Fuente: elaboración propia. 
La variable dependiente tomada ha sido el recuerdo de voto para las elecciones generales de 2015 y 2016 (“¿Podría decirme el nombre del partido o coalición por el cual votó Ud. en las generales del pasado... ?”, pregunta 31 en ambos cuestionarios) para cada uno de los cinco principales partidos nacionales (PP, PSOE, Ciudadanos, Podemos e Izquierda Uni$\left.\mathrm{da}^{3}[\mathrm{IU}]\right)$ así como para los dos principales partidos independentistas de Cataluña: Esquerra Republicana de Catalunya (ERC) y Convergència. ${ }^{4} \mathrm{El}$ objetivo es obtener las explicaciones a nivel individual (y no de manera agregada) a los efectos del consumo informativo.

Las variables independientes introducidas en nuestro modelo de estudio han sido el consumo mediático de información política de los ciudadanos durante la campaña electoral de cada uno de los medios de comunicación (pregunta 19 del estudio n. ${ }^{\circ} 3126$ y 20 del estudio n. ${ }^{\circ} 3145$ ), al menos de los más mayoritarios (y con mayor muestra estadística): en prensa, El País, El Mundo, ABC, La Razón, Público, periódicos digitales; en radio, Cadena Ser, Cadena Cope, Onda Cero y RNE; y en televisión, TVE1, TVE2, Antena 3, Cuatro, Telecinco y La Sexta. Con el fin de medir la polarización mediática de la opinión pública en Cataluña, se han tomado medios de esta comunidad: en prensa, El Periódico de Catalunya y La Vanguardia; en radio, RAC1; y en televisión, TV3.

Por último, como variables de control, se han introducido las variables políticamente relevantes y de carácter sociodemográfico: sexo, edad, estatus socioeconómico ${ }^{5}$ (clase alta/media-alta, nuevas clases medias, viejas medias, obreros cualificados, obreros no cualificados), percepción de la situación política y económica actual (siendo 1 muy buena a 5 muy mala) y la ideología (1, izquierda; 10, derecha).

\section{Hipótesis}

La revisión bibliográfica expuesta en los apartados anteriores nos ha permitido formular las siguientes hipótesis de trabajo:

\footnotetext{
En coalición electoral con Podemos en 2016 bajo la fórmula Unidos Podemos.

Convergència-Democràcia i Llibertat.

Las categorías de clasificación del estatus socioeconómico han sido las incorporadas por el Centro de Investigaciones Sociológicas (CIS, s. f.) en función de varios criterios, como renta familiar, ocupación, actividad económica, etc.
} 
- Hipótesis 1 (H1): El consumo mediático tendrá consecuencias desde el punto de vista de la movilización y conversión de las preferencias electorales de los individuos.

- Hipótesis 2 (H2): La movilización o conversión del voto dependerá de la selección que realizan los electores.

- Hipótesis 3 (H3): La selección estará condicionada por la afinidad ideológica con el medio.

- Hipótesis 4 (H4): Será en la prensa en la que se darán los mayores efectos en el voto de la selección informativa de los electores.

\section{Resultados}

\section{Estudio de caso 1: elecciones generales de diciembre de 2015}

En las elecciones generales de 2015, el sistema político de nuestro país se enfrentaba por primera vez a la irrupción a nivel nacional de dos nuevos partidos que habían cosechado un éxito notable en las elecciones al Parlamento Europeo de 2014: Podemos y Ciudadanos. Esta irrupción supuso un cambio en nuestro sistema mediático bipartidista que tuvo que adaptarse a ese nuevo escenario y ampliar el espacio informativo a nuevos partidos, al igual que en sus líneas editoriales. El consumo mediático de 2015 se centró en dos pilares: la alineación partidista y la polarización mediática (tabla 3).

En el PP, observamos una presencia más significativa de medios de comunicación dentro del espectro conservador o liberal. En prensa, prefieren $E l M u n d o$ y el $A B C$, y son poco proclives a usar prensa digital. En radios, utilizan Cadena Cope (conservadora) y Onda Cero (centro-liberal), y usan más que la media este canal. En televisión, son más cercanos a la cadena pública y Antena 3, si bien entre las televisiones, el sesgo ideológico es más complicado de determinar. Pues bien, su consumo mediático influyó de forma clara en el voto: los votantes que se informan a través de periódicos nacionales como ABC, La Razón y, en menor medida, El Mundo, presentan una mayor probabilidad de votar a este partido; efecto contrario que hemos observado en el caso de El País. No observamos incidencias claras en televisión o radio, pese al mayor consumo de Onda Cero o Cadena Cope. 
La incidencia de factor ideológico es muy alta (electores con autoubicación más a la derecha [7-10] muestran una mayor probabilidad del voto al PP).

Tabla 3. Consumo mediático según voto y medio en la campaña electoral de 2015

\begin{tabular}{|c|c|c|c|c|c|c|c|}
\hline & PP & PSOE & Podemos $^{\mathrm{a}}$ & Ciudadanos & IU & ERC & Convergéncia ${ }^{\mathrm{b}}$ \\
\hline \multicolumn{8}{|l|}{ Prensa } \\
\hline El País & $7,3 \%$ & $36,7 \%$ & $27,7 \%$ & $19,3 \%$ & $34,5 \%$ & $2,5 \%$ & $3,1 \%$ \\
\hline El Mundo & $19,7 \%$ & $3,2 \%$ & $3 \%$ & $21,7 \%$ & $4,2 \%$ & $0 \%$ & $0 \%$ \\
\hline$A B C$ & $14,2 \%$ & $2 \%$ & $0,6 \%$ & $3,2 \%$ & $1,7 \%$ & $0 \%$ & $0 \%$ \\
\hline La Razón & $5,6 \%$ & $1 \%$ & $0,2 \%$ & $2,1 \%$ & $1,7 \%$ & $0 \%$ & $0 \%$ \\
\hline Público & $0,2 \%$ & $0 \%$ & $6,6 \%$ & $0 \%$ & $4,2 \%$ & $0 \%$ & $0 \%$ \\
\hline $\begin{array}{l}\text { Periódicos } \\
\text { digitales }\end{array}$ & $1,7 \%$ & $1 \%$ & $8,1 \%$ & $3,5 \%$ & $10,1 \%$ & $4,2 \%$ & $0 \%$ \\
\hline La Vanguardia & $2,4 \%$ & $3,9 \%$ & $4,5 \%$ & $3,8 \%$ & $0 \%$ & $26,3 \%$ & $36,9 \%$ \\
\hline $\begin{array}{l}\text { El Periódico de } \\
\text { Catalunya }\end{array}$ & $0,6 \%$ & $4,2 \%$ & $4,4 \%$ & $1,6 \%$ & $0 \%$ & $25,4 \%$ & $15,4 \%$ \\
\hline \multicolumn{8}{|l|}{ Radio } \\
\hline Cadena Ser & $11,9 \%$ & $54,4 \%$ & $55,4 \%$ & $17,5 \%$ & $64,9 \%$ & $3,8 \%$ & $4,7 \%$ \\
\hline Cadena Cope & $39,1 \%$ & $6,1 \%$ & $4,5 \%$ & $21,4 \%$ & $7,2 \%$ & $0 \%$ & $0 \%$ \\
\hline Onda Cero & $16,9 \%$ & $10,3 \%$ & $9,7 \%$ & $21,8 \%$ & $10,3 \%$ & $1,9 \%$ & $0 \%$ \\
\hline RNE & $11,6 \%$ & $10,3 \%$ & $8,1 \%$ & $12,3 \%$ & $5,2 \%$ & $1,9 \%$ & $0 \%$ \\
\hline RAC1 & $0 \%$ & $0,9 \%$ & $3,2 \%$ & $0,3 \%$ & $0 \%$ & $47,1 \%$ & $34,9 \%$ \\
\hline \multicolumn{8}{|l|}{ Televisión } \\
\hline TVE1 & $35,6 \%$ & $19,6 \%$ & $6,3 \%$ & $16,6 \%$ & $9,7 \%$ & $1,8 \%$ & $5,1 \%$ \\
\hline TVE2 & $0,3 \%$ & $0,2 \%$ & $0,3 \%$ & $0,3 \%$ & $0,6 \%$ & $0 \%$ & $0 \%$ \\
\hline Antena 3 & $25 \%$ & $14,9 \%$ & $10,2 \%$ & $32,7 \%$ & $12,5 \%$ & $2,5 \%$ & $2 \%$ \\
\hline Cuatro & $1,6 \%$ & $8 \%$ & $8,7 \%$ & $6,6 \%$ & $7,4 \%$ & $0,6 \%$ & $3,1 \%$ \\
\hline Telecinco & $11,1 \%$ & $18,7 \%$ & $8,4 \%$ & $12,7 \%$ & $8 \%$ & $1,8 \%$ & $5,1 \%$ \\
\hline La Sexta & $3,2 \%$ & $21,5 \%$ & $51,3 \%$ & $16,6 \%$ & $48,3 \%$ & $8 \%$ & $1 \%$ \\
\hline TV3 & $0,4 \%$ & $1,8 \%$ & $3,7 \%$ & $1,4 \%$ & $0 \%$ & $76,7 \%$ & $74,5 \%$ \\
\hline
\end{tabular}

a Incluye Podemos, En Comú Podem, Compromís-Podemos-És el Moment y En Marea.

${ }^{\mathrm{b}}$ Convergència-Democràcia i Llibertat.

Fuente: elaboración propia según encuesta poselectoral del CIS n. ${ }^{0} 3126$. 
En los votantes del PSOE, observamos un consumo informativo de centro y centro-izquierda: El País y Cadena Ser, así como escaso uso de medios digitales y de prensa y radios conservadores. En televisión, destaca La Sexta (de tendencia progresista), Telecinco (sin ideología clara) o la cadena pública, Televisión Española (TVE), pero con una distribución más pareja. Los efectos en el voto no son tan profundos como en el caso de los votantes del PP: existen mayores incidencias en cuestiones sociodemográficas que mediáticas (un electorado formado por una mayor proporción de mujeres, por electores de mayor edad y por clases sociales trabajadoras - obreros cualificados y no cualificados-, de ahí la menor penetración de lectura de prensa escrita observada en los análisis previos). El País es el medio con mayor incidencia probabilística en influencia en el voto de los electores socialistas, así como efectos negativos con El Mundo y periódicos digitales (muy en consonancia con el envejecimiento de la población y la feminización de su electorado, y la brecha digital formada en torno a estos dos perfiles poblacionales).

En Podemos e IU, que muestran comportamientos similares, la tendencia es al uso de medios de comunicación de izquierda y poca permeabilización de medios conservadores (mayor incidencia que el resto, un $10 \%$ de IU o un $8 \%$ de Podemos, frente al $1 \%$ de los votantes que usan este medio por PSOE o PP). Además, muestran menor confianza en las cadenas públicas (RNE y TVE), y prefieren Cadena Ser o El País (en menor medida que los votantes del PSOE), así como, en televisión, La Sexta.

Las regresiones muestran que Podemos posee un electorado sociodemográfico estadísticamente más joven y muy masculinizado (en la misma proporción que el PSOE, muestra un perfil de votantes formado por más mujeres). Además, se detalla un posicionamiento ideológico escorado hacia la izquierda (1-3 dentro del espectro), de lo que derivan sus filiaciones mediáticas. Aquellos votantes que se informan a través de la prensa progresista, Público especialmente, muestran mayores probabilidades de votar a este partido; variable relacionada con la edad de su electorado (formado especialmente por menores de 35 años), de ahí su fuerte relación entre el consumo de prensa digital y voto. 
Similares características evidencia el electorado de IU, pese a que la muestra es menor.

Los votantes de Ciudadanos no manifiestan preferencias claras por ningún medio, si bien tienden a medios ligeramente conservadores. No existen claras referencias en prensa, pero El País y El Mundo son los medios con mayor repercusión. En radio, se dividen casi a partes iguales, entre Cadena Ser, Cadena Cope y Onda Cero; y en televisión, muestran una mayor predisposición a Antena 3, pero sin una tendencia clara hacia ningún medio.

Las relaciones entre voto y consumo del electorado de Ciudadanos muestran un perfil conservador, pero más moderado que en el caso del PP. Su dieta informativa más plural muestra relaciones estadísticas muy dispares: observamos mayores probabilidades en el voto a Ciudadanos en aquellos lectores que consumen prensa conservadora ( $E l$ Mundo) como progresista (Cadena Ser), y menores probabilidades de voto en aquellos que leen prensa de perfil más conservador, como el caso de $A B C$.

En los electores nacionalistas catalanes (ERC y Convergència), observamos un consumo unidireccional muy segregado hacia medios autonómicos, especialmente del canal público de la Generalitat, pero también prensa local (El periódico de Catalunya y La Vanguardia).

Más allá de las variables sociodemográficas (el perfil de Convergència/Democràcia i Llibertat muestra un electorado de mayor edad), se observa un consumo mediático muy endémico, lo que repercute en el voto hacia estas formaciones. Sin considerar las cuestiones ideológicas, la lectura de prensa territorial es la que mayor efecto tiene sobre la posibilidad de votar a estas formaciones, si bien la relación es mucho más fuerte en los votantes de ERC por prensa más progresista (El Periódico de Catalunya) y los votantes conservadores de Convergència por La Vanguardia (de perfil centro-derecha). La exposición a la radio catalana (RAC1) y a la televisión pública autonómica (TV3) aumenta la relación con el voto hacia estos dos partidos, incluso, en los votantes de ERC, cuya relación estadística es inversa: la no exposición a televisiones nacionales aumenta su probabilidad en el voto (tabla 4). 


\section{Tabla 4. Efectos estadísticos entre el consumo mediático y el voto en 2015}

\begin{tabular}{|c|c|c|c|c|c|c|c|}
\hline & PP & PSOE & Podemos $^{\mathrm{a}}$ & Ciudadanos & $\mathrm{IU}^{\mathrm{b}}$ & ERC & Convergéncia $^{c}$ \\
\hline Sexo & $-0,004$ & $0,301^{* * *}$ & $-0,366^{* * *}$ & $-0,079$ & 0,026 & $-0,132$ & $-0,063$ \\
\hline Edad & $0,016^{* * *}$ & $0,022^{* * *}$ & $-0,027^{* * *}$ & $-0,033^{* * *}$ & 0,002 & $-0,003$ & $0,021^{* * *}$ \\
\hline \multicolumn{8}{|l|}{ Estatus socioeconómico } \\
\hline Clase alta/media-alta & 0,290 & $-0,045$ & 0,499 & $-0,161$ & $-0,043$ & 0,252 & 16,070 \\
\hline Nuevas clases medias & 0,170 & 0,374 & $0,539^{*}$ & $-0,082$ & $-0,027$ & $-0,628$ & 16,434 \\
\hline Viejas clases medias & $0,611^{*}$ & 0,479 & 0,199 & $-0,376$ & $-0,007$ & 0,436 & 16,689 \\
\hline Obreros cualificados & 0,326 & $0,917^{* * *}$ & 0,523 & $0,571^{* *}$ & $-0,231$ & $-0,369$ & 16,567 \\
\hline Obreros no cualificados & 0,356 & $0,958^{* * *}$ & $0,596^{*}$ & $-0,543^{*}$ & $-0,217$ & $-0,717$ & 15,962 \\
\hline Percepción política actual & $-0,088$ & $-0,086^{*}$ & $-0,088$ & $0,159^{* * *}$ & $-0,084$ & 0,237 & 0,064 \\
\hline $\begin{array}{l}\text { Percepción económica } \\
\text { actual }\end{array}$ & $-0,418^{* * *}$ & $-0,067$ & $0,291^{* * *}$ &,$- 0189^{* * *}$ & 0,080 & 0,262 & $-0,241$ \\
\hline Ideología (1-10) & $1,053^{* * *}$ & $-0,376^{* * *}$ & $-0,602^{* * *}$ & $0,300^{* * *}$ & $-0,530^{* * *}$ & $-0,516^{* * *}$ & $0,286^{* * *}$ \\
\hline \multicolumn{8}{|l|}{ Prensa } \\
\hline El País & $-0,581^{* * *}$ & $0,502^{* * *}$ & $0,397^{* * *}$ & 0,214 & $0,636^{* * *}$ & $-1,567^{* * *}$ & $-0,577$ \\
\hline El Mundo & $0,446^{* *}$ & $-0,763^{* * *}$ & $-0,371$ & $0,804^{* * *}$ & 0,305 & $-15,922$ & $-16,231$ \\
\hline$A B C$ & $1,112^{* * *}$ & $-0,324$ & $-0,848$ & $-0,747^{* *}$ & 0,296 & $-15,217$ & $-16,609$ \\
\hline La Razón & $0,689^{*}$ & $-0,467$ & $-1,370$ & $-0,198$ & 0,807 & $-15,550$ & $-16,327$ \\
\hline Público & $-0,051$ & $-19,818$ & $2,868^{* * *}$ & $-18,758$ & 0,618 & $-17,457$ & $-14,948$ \\
\hline Periódicos digitales & $-0,013$ & $-1,633^{* * *}$ & $1,217^{* * *}$ & $-0,211$ & $1,042^{* * *}$ & 0,275 & 16,046 \\
\hline La Vanguardia & - & - & - & - & - & 0,538 & $1,353^{* * *}$ \\
\hline El Periódico de Catalunya & - & - & - & - & - & $1,121^{* * *}$ & 0,660 \\
\hline \multicolumn{8}{|l|}{ Radio } \\
\hline Cadena Ser & $-0,152$ & $-0,361^{* * *}$ & $-0,137$ & $0,314^{* * *}$ & 0,176 & $0,579^{* *}$ & $-0,685^{*}$ \\
\hline Cadena Cope & 0,041 & $-0,353$ & 0,221 & 0,111 & $0,957^{* * *}$ & 0,687 & 0,530 \\
\hline Onda Cero & 0,039 & $-0,250$ & $0,346^{*}$ & 0,082 & 0,957 & $-0,197$ & $-0,657$ \\
\hline RNE & 0,056 & $-0,096$ & $-0,496$ & 0,067 & $1,143^{* *}$ & $-0,645$ & $-17,114$ \\
\hline RAC1 & - & - & - & - & - & $1,792^{* * *}$ & 0,152 \\
\hline \multicolumn{8}{|l|}{ Televisión } \\
\hline TVE1 & $-0,731$ & $-0,085$ & $-0,329$ & 0,634 & 17,479 & $-2,603^{* *}$ & 16,018 \\
\hline TVE2 & $-0,849$ & 0,128 & $-0,593$ & 0,750 & 17,393 & $-2,691^{* *}$ & 15,635 \\
\hline Antena 3 & $-1,318$ & 0,014 & 0,762 & 0,815 & 17,717 & $-2,432^{* *}$ & 15,973 \\
\hline Cuatro & $-0,823$ & 0,119 & $-0,159$ & 0,036 & 15,816 & $-18,544$ & 0,444 \\
\hline Telecinco & $-0,999$ & $-0,154$ & $-0,677$ & 0,017 & 17,878 & $-2,712^{* *}$ & 16,044 \\
\hline La Sexta & $-1,176$ & $-0,449$ & $-0,899$ & 0,073 & 18,001 & $-1,684$ & 16,912 \\
\hline TV3 & & - & - & - & - & $3,517^{* * *}$ & $4,216^{* * *}$ \\
\hline Constante & $-5,648$ & $-1,304$ & 1,968 & $-2,169$ & $-19,126$ & $-1,912$ & $-39,357$ \\
\hline Chi cuadrada & 2413,175 & 617,609 & 1211,830 & 433,056 & 208,973 & 775,274 & 385,597 \\
\hline $\begin{array}{l}-2 \log \text { de verosimilitud } \\
\text { final }\end{array}$ & 2976,363 & 4497,518 & 4027,815 & 3434,577 & 1482,870 & 757,274 & 611,266 \\
\hline R cuadrado de Cox y Snell & 0,362 & 0,109 & 0,202 & 0,077 & 0,038 & 0,134 & 0,069 \\
\hline R cuadrado de Nagelkerke & 0,571 & 0,177 & 0,324 & 0,151 & 0,141 & 0,541 & 0,409 \\
\hline
\end{tabular}

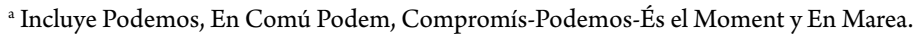

${ }^{\mathrm{b}}$ Muestra muy reducida.

${ }^{\mathrm{c}}$ Convergència-Democràcia i Llibertat.

Fuente: elaboración propia según encuesta poselectoral del CIS n. ${ }^{\circ} 3126$. 


\section{Estudio de caso 2: elecciones generales de junio de 2016}

En las elecciones generales de 2016, el escenario construido durante los meses previos posibilitaba la polarización creciente de la opinión pública. Las posiciones de los partidos, cada vez más radicalizadas sobre la acción realizada durante el periodo entre los dos comicios, se trasladaron a los medios de comunicación.

En lo referido a nuestro modelo de estudio, las primeras tendencias que apuntamos se reproducen de la misma manera que en el caso anterior, si bien con una menor exposición mediática. El hastío provocado por esa polarización partidista recogido por los medios de comunicación se trasladó a una menor participación en los comicios (tabla 5).

Tabla 5. Consumo mediático según voto y medio en la campaña electoral de 2016

\begin{tabular}{|c|c|c|c|c|c|c|}
\hline & PP & PSOE & $\begin{array}{l}\text { Unidos } \\
\text { Podemos }^{\mathrm{a}}\end{array}$ & Ciudadanos & ERC & Convergència \\
\hline \multicolumn{7}{|l|}{ Prensa } \\
\hline El País & $8,1 \%$ & $38,2 \%$ & $26,1 \%$ & $21,8 \%$ & $2,2 \%$ & $0 \%$ \\
\hline El Mundo & $21,7 \%$ & $4,2 \%$ & $3,9 \%$ & $19,2 \%$ & $0 \%$ & $0 \%$ \\
\hline$A B C$ & $11,1 \%$ & $1,5 \%$ & $0,2 \%$ & $3,8 \%$ & $0 \%$ & $0 \%$ \\
\hline La Razón & $4,2 \%$ & $0,2 \%$ & $0,2 \%$ & $1,9 \%$ & $0 \%$ & $0 \%$ \\
\hline Público & $0,4 \%$ & $0,7 \%$ & $6 \%$ & $0 \%$ & $0 \%$ & $0 \%$ \\
\hline Periódicos digitales & $2,1 \%$ & $2,2 \%$ & $10,5 \%$ & $3,1 \%$ & $1,1 \%$ & $0 \%$ \\
\hline La Vanguardia & $0,6 \%$ & $3,2 \%$ & $3,9 \%$ & $4,2 \%$ & $29,7 \%$ & $34,2 \%$ \\
\hline El Periódico de Catalunya & $0,8 \%$ & $4 \%$ & $6,8 \%$ & $4,2 \%$ & $9,9 \%$ & $13,2 \%$ \\
\hline \multicolumn{7}{|l|}{ Radio } \\
\hline Cadena Ser & $8,8 \%$ & $57,7 \%$ & $56 \%$ & $24,4 \%$ & $3,6 \%$ & $3,3 \%$ \\
\hline Cadena Cope & $43,5 \%$ & $6 \%$ & $4,5 \%$ & $18,2 \%$ & $0 \%$ & $0 \%$ \\
\hline Onda Cero & $15,9 \%$ & $10,1 \%$ & $6 \%$ & $21,6 \%$ & $0 \%$ & $3,3 \%$ \\
\hline RNE & $11,7 \%$ & $10,1 \%$ & $10,3 \%$ & $9,1 \%$ & $0 \%$ & $0 \%$ \\
\hline \multicolumn{7}{|l|}{ RAC1 } \\
\hline \multicolumn{7}{|l|}{ Televisión } \\
\hline TVE1 & $32,9 \%$ & $17,4 \%$ & $9,3 \%$ & $20,9 \%$ & $0,8 \%$ & $0 \%$ \\
\hline TVE2 & $0,5 \%$ & $0,1 \%$ & $0,6 \%$ & $0 \%$ & $0 \%$ & $0 \%$ \\
\hline Antena 3 & $22,7 \%$ & $16,4 \%$ & $10 \%$ & $28,7 \%$ & $0,8 \%$ & $3,7 \%$ \\
\hline Cuatro & $2,6 \%$ & $7,3 \%$ & $7,7 \%$ & $8 \%$ & $0 \%$ & $1,9 \%$ \\
\hline Telecinco & $13,8 \%$ & $21,8 \%$ & $8,9 \%$ & $13,6 \%$ & $2,5 \%$ & $0 \%$ \\
\hline La Sexta & $4,2 \%$ & $23,5 \%$ & $50,7 \%$ & $17,1 \%$ & $3,4 \%$ & $0 \%$ \\
\hline TV3 & $0,6 \%$ & $1,2 \%$ & $4,7 \%$ & $1,8 \%$ & $80,5 \%$ & $74,1 \%$ \\
\hline
\end{tabular}

a Incluye Podemos, IU, En Comú Podem, Compromís-Podemos-És el Moment y En Marea.

Fuente: elaboración propia según encuesta postelectoral del CIS n. ${ }^{0} 3145$. 
Por recuerdo de voto, el consumo mediático muestra similares tendencias a lo observado en las elecciones de diciembre de 2015, de ahí que nos centremos en la influencia de esta dieta informativa en el voto. Tal y como se aprecia en la tabla 6 , podemos confirmar las tendencias citadas en lo referido a consumo de medios.

Deberíamos preguntarnos en qué partidos hay una mayor incidencia del consumo mediático y, en ese sentido, es en el PP y en Podemos en los que tenemos una mayor exposición y mayores efectos explicativos de voto. En cambio, en Ciudadanos y en el PSOE es menor, quizá, porque las explicaciones de voto están más allá de estas variantes, más cercanas a cuestiones sociodemográficas (escuela de Columbia) o de afiliaciones partidistas (escuela de Míchigan).

A diferencia del estudio de caso anterior, observamos una mayor incidencia de la televisión como medio de influencia en el voto como técnica de reafirmación. La influencia en el electorado de los partidos más sistémicos (PP, PSOE y Ciudadanos) es más débil que en el caso de Podemos/ IU, aun observando preferencias ideológicas (Televisión Española, ambas, para el caso del PP; La Sexta, para el PSOE; y Antena 3, para Ciudadanos). En el partido más outsider, Podemos, sí que observamos mayor probabilidad de voto con La Sexta como refuerzo de sus predisposiciones políticas.

Pese a ello, observamos una mayor probabilidad de voto con rechazos informativos, como en el caso del PP con La Sexta y con los votantes de los partidos nacionalistas catalanes con medios centralistas, especialmente en ERC.

Es significativo cómo el eje de discusión en Cataluña infiere en el consumo de los votantes de los partidos independentistas catalanes. Este consumo endogámico que apreciamos en las elecciones generales de 2015, y que se repercutió en el consumo televisivo, vuelve a reproducirse en la radio en 2016. Mientras que en los votantes de los partidos nacionales el eje de actuación y consumo-refuerzo es el ideológico, en estos casos es el territorial, de modo que es la radio pública la de mayor impacto en sus electores. 


\section{Tabla 6. Efectos estadísticos entre el consumo mediático y el voto en 2016}

\begin{tabular}{|c|c|c|c|c|c|c|}
\hline & PP & PSOE & $\begin{array}{c}\text { Unidos } \\
\text { Podemos }^{\mathrm{a}}\end{array}$ & Ciudadanos & ERC & Convergència \\
\hline Sexo & $0,179^{*}$ & $0,215^{* * *}$ & $-0,153^{*}$ & 0,043 & $-0,503^{* *}$ & $-0,053$ \\
\hline Edad & $0,018^{* * *}$ & $0,025^{* * *}$ & $-0,026^{* * *}$ & $-0,023^{* * *}$ & 0,007 & $0,018^{* *}$ \\
\hline \multicolumn{7}{|l|}{ Estatus socioeconómico } \\
\hline Clase altas/media-alta & 0,517 & 0,287 & $-0,097$ & 0,402 & 0,976 & $-1,162^{*}$ \\
\hline Nuevas clases medias & $0,549^{*}$ & $0,639^{* *}$ & 0,048 & 0,446 & 0,128 & $-0,974$ \\
\hline Viejas clases medias & $0,998^{* * *}$ & 0,483 & $-0,185$ & $-0,037$ & 0,197 & $-1,461^{* *}$ \\
\hline Obreros cualificados & $0,703^{* *}$ & $1,101^{* * *}$ & $-0,323$ & $-0,060$ & $-0,307$ & $-0,930$ \\
\hline Obreros no cualificados & 0,389 & $1,023^{* * *}$ & $-0,279$ & $-0,068$ & 0,446 & $-1,676^{*}$ \\
\hline Percepción política actual & $-0,156^{* *}$ & $-0,194^{* * *}$ & 0,080 & $0,127^{* * *}$ & 0,175 & $0,494^{*}$ \\
\hline Percepción económica actual & $-0,512^{* * *}$ & 0,001 & $0,219^{* * *}$ & $-0,078$ & $-0,010$ & $-0,299$ \\
\hline Ideología (1-10) & $1,006^{* * *}$ & $-0,397^{* * *}$ & $-0,687^{* * *}$ & $0,258^{* * *}$ & $-0,524^{* * *}$ & $0,253^{* * *}$ \\
\hline \multicolumn{7}{|l|}{ Prensa } \\
\hline El País & $-0,473^{* * *}$ & $0,624^{* * *}$ & 0,056 & $0,417^{* *}$ & $-1,625$ & $-15,203$ \\
\hline El Mundo & $0,490^{* * *}$ & $-0,434$ & $-0,063$ & $0,633^{* * *}$ & $-14,639$ & $-14,923$ \\
\hline$A B C$ & $0,893^{* * *}$ & $-0,260$ & $-1,632$ & 0,158 & $-14,863$ & $-15,898$ \\
\hline La Razón & 0,265 & $-1,206$ & $-0,871$ & 0,097 & $-13,074$ & $-15,737$ \\
\hline Público & 1,062 & $-1,326^{* *}$ & $1,136^{* * *}$ & $-18,622$ & $-13,074$ & $-14,270$ \\
\hline Periódicos digitales & $-0,754$ & $-1,013^{* * *}$ & $1,152^{* * *}$ & $-0,322$ & 0,044 & $-15,235$ \\
\hline La Vanguardia & - & - & - & - & 0,517 & $0,842^{* *}$ \\
\hline El Periódico de Catalunya & - & - & - & 一 & 0,299 & 0,805 \\
\hline \multicolumn{7}{|l|}{ Radio } \\
\hline Cadena Ser & $-0,610^{* * *}$ & $0,343^{* * *}$ & $0,259^{*}$ & $-0,144$ & $-0,738$ & $-0,207$ \\
\hline Cadena Cope & $0,529^{* * *}$ & $-0,653^{* *}$ & 0,111 & $-0,339$ & $-14,841$ & 15,818 \\
\hline Onda Cero & 0,178 & 0,072 & $-0,201$ & $0,519^{* * *}$ & $-16,075$ & 0,115 \\
\hline RNE & 0,069 & $-0,047$ & $0,558^{* *}$ & 0,072 & $-16,949$ & $-15,529$ \\
\hline $\mathrm{RAC1}$ & - & - & - & - & $1,395^{* * *}$ & $0,899^{* *}$ \\
\hline \multicolumn{7}{|l|}{ Televisión } \\
\hline TVE1 & $0,715^{* * *}$ & $0,613^{* * *}$ & 0,160 & $0,428^{* * *}$ & $-2,054^{* *}$ & $-16,451$ \\
\hline TVE2 & $1,975^{* * *}$ & $-1,397$ & $1,298^{*}$ & $-18,163$ & $-17,128$ & $-15,671$ \\
\hline Antena 3 & $0,592^{* * *}$ & $0,737^{* * *}$ & 0,061 & $0,792^{* * *}$ & $-2,176^{* *}$ & $-1,212$ \\
\hline Cuatro & $-0,142$ & $0,791^{* * *}$ & $0,788^{* * *}$ & $0,933^{* * *}$ & $-16,995$ & $-0,488$ \\
\hline Telecinco & $0,398^{* * *}$ & $1,072^{* * *}$ & $-0,042$ & $0,470^{* * *}$ & $-1,443^{*}$ & $-16,550$ \\
\hline La Sexta & $-0,651^{* * *}$ & $0,376^{* * *}$ & $1,332^{* * *}$ & $0,415^{* *}$ & $-1,826^{* * *}$ & $-15,885$ \\
\hline TV3 & - & - & - & - & $2,373^{* * *}$ & $2,487^{* * *}$ \\
\hline Constante & $-6,554$ & $-1,831$ & 1,060 & $-3,437$ & $-2,370$ & $-6,355$ \\
\hline Chi cuadrada & 2513,181 & 735,940 & 1530,908 & 289,401 & 611,772 & 229,034 \\
\hline$-2 \log$ de verosimilitud final & 2706,288 & 4341,041 & 3449,312 & 2811,864 & 613,908 & 387,830 \\
\hline R cuadrado de Cox y Snell & 0,389 & 0,134 & 0,259 & 0,055 & 0,113 & 0,044 \\
\hline R cuadrado de Nagelkerke & 0,607 & 0,213 & 0,416 & 0,121 & 0,529 & 0,385 \\
\hline
\end{tabular}

${ }^{a}$ Incluye Unidos Podemos, En Comú Podem, Compromís-Podemos-EUPV y En Marea.

Fuente: elaboración propia según encuesta poselectoral del CIS n. ${ }^{\circ} 3145$. 
Además, se reproducen las mismas relaciones en la prensa, especialmente en los votantes de Convergència. Nuevamente, el consumo de prensa más influyente en el voto en las formaciones autonómicas es la prensa autonómica (La Vanguardia y El Periódico de Catalunya), que poseen una línea informativa más cercana a sus presupuestos políticos.

\section{Conclusiones}

En este artículo, hemos pretendido observar los efectos políticos de los medios de comunicación en la opinión pública española, más concretamente, la exposición selectiva y su influencia en el voto en dos estudios de caso (las elecciones generales de 2015 y 2016 en España). El aumento de la polarización y politización de los sistemas mediáticos afecta intensamente al buen funcionamiento de las democracias y a la salud de los medios de comunicación como vigilantes del poder.

La principal consecuencia observada es que los votantes realizan una selección entre los diferentes medios y enfoques periodísticos que le son más próximos y coherentes con sus opiniones. Estos criterios de selección, constatados en los modelos estadísticos aplicados, no son puramente ideológicos, sino también territoriales dentro de un segundo eje de competición (centro-periferia), y más en concreto, en su posición acerca de la independencia de la región de Cataluña. Esto provoca una fuerte polarización de los votantes en el consumo de medios, con rechazos mediáticos muy fuertes a consumir medios o canales ajenos a su postura ideológica y reduciendo la variedad de fuentes de su dieta informativa, como un refuerzo de sus posiciones partidistas (Stroud, 2010), tal y como expresábamos en las hipótesis $\mathrm{H} 1, \mathrm{H} 2$ y H3.

La evidencia empírica más reseñable en los dos casos de estudio analizados es que la exposición selectiva de los votantes actúa como factor de refuerzo frente a la conversión. Si los electores priorizan visiones coherentes con sus opiniones y reducen su variedad de mensajes en su dieta informativa, la probabilidad de cambiar de opción electoral disminuye. A esto tenemos que sumar la escasa permeabilidad de los votantes a usar medios poco afines e, incluso, el rechazo cada vez más pronunciado hacia medios contrarios o no alineados con su postura política (ideológica o territorial). 
La investigación ha demostrado evidencia sobre la influencia del consumo mediático de prensa (hipótesis $\mathrm{H} 4$ ) en el voto debido al marcado perfil ideológico que este medio posee. La televisión, en cambio, es en líneas generales la que menor relevancia estadística posee debido a que la menor carga editorial (con respecto a la prensa o la radio) impide a los votantes activar los mecanismos mentales de selección de contenidos, pese a que encontremos algunas excepciones como la influencia de los votantes de Podemos con La Sexta, debido a su definido perfil progresista.

Aun así, hemos de advertir al lector varias limitaciones de esta investigación. El enfoque cuantitativo de la encuesta del organismo español CIS impide analizar el papel de los filtros interpretativos de los votantes con la prensa digital y las redes sociales, y más cuando la propia encuesta reconoce que cada vez una mayor proporción de la población se informa políticamente a través de esta plataforma. Para ello, se requieren estudios cualitativos y otras formas de medición cuantitativa que se escapan a este estudio. Además, la ausencia de prensa digital dentro de la codificación de la encuesta (en concreto, de qué cabeceras) también nos impide analizar el sistema mediático en su conjunto y, más en detalle, la exposición selectiva y su comportamiento en el voto de los más jóvenes, más propensos a utilizar estas herramientas para informarse de la actualidad política.

Por ello, debemos poner en cautela los resultados y analizarlos dentro de las tendencias que vemos trazadas en el sistema mediático español, en el que cada vez la prensa impresa y la radio tiene una menor penetración, y las redes sociales y la prensa digital ejercen una mayor influencia . Los estudios sobre los efectos en el voto en las elecciones generales de abril de 2019 (con la llegada de un partido de derecha radical populista, VOX) nos permitirán seguir ahondando en el papel de los medios en su función como movilizador o conversor de las preferencias electorales de los ciudadanos.

\section{Referencias}

Anduiza, E. y Bosch, A. (2012). Comportamiento político y electoral. Ariel. 
Arendt, F. (2013). Dose-dependent media priming effects of stereotypic newspaper articles on implicit and explicit stereotypes. Journal of Communication, 63(5), 830-851. https://doi.org/10.1111/ jcom. 12056

Arendt, F., Northup, T. \& Camaj, L. (2019). Selective exposure and news media brands: Implicit and explicit attitudes as predictors of news choice. Media Psychology, 22(3), 526-543. https://doi.org/10.10 80/15213269.2017.1338963

Arendt, F., Steindl, N. \& Kümpel, A. (2016). Implicit and explicit attitudes as predictors of gatekeeping, selective exposure, and news sharing: Testing a general model of media-related selection. Journal of Communication, 66(5), 717-740. https://doi.org/10.1111/jcom.12256

Bardin, B., Vidal, P., Facca, L., Dumas, R. \& Perrissol, S. (2018). The effect of information quality evaluation on selective exposure in informational cognitive dissonance: The role of information novelty. International Review of Social Psychology, 31(1). http://doi. org/10.5334/irsp. 173

Bennett, W. L. \& Iyengar, S. (2008). A new era of minimal effects? The changing foundations of political communication. Journal of Communication, 58(4), 707-731. https://doi.org/10.1111/j.14602466.2008.00410.x

Botero, S., Cornejo, R. C., Gamboa, L., Pavão, N. \& Nickerson, D. W. (2019). Under friendly fire: An experiment on partisan press, fragmented opposition and voting behavior. Electoral Studies, 60, 102044. https://doi.org/10.1016/j.electstud.2019.04.008

Bou-Hamad, I. \& Yehya, N. A. (2016). Partisan selective exposure in TV consumption patterns: A polarized developing country context. Communication Research, 47(1), 55-81. https://doi. org/10.1177/0093650216681896 
Campbell, A., Converse, P., Miller, W. y Stokes, D. (1960). The american voter. John Wiley \& Sons.

Centro de Investigaciones Sociológicas. (s. f.). Nota de investigación: actualización de las clasificaciones nacionales de ocupación y de actividad económica en los datos del CIS. https://www.cis.es/cis/export/sites/default/-Archivos/NotasdeInvestigacion/NI010_CNO11CNAE09_Informe.pdf

Chaffee, S. H. \& Miyo, Y. (1983). Selective exposure and the reinforcement hypothesis: An intergenerational panel study of the 1980 presidential campaign. Communication Research, 10(1), 3-36. https://doi. org/10.1177/009365083010001001

Dilliplane, S. (2014). Activation, conversion, or reinforcement? The impact of partisan news exposure on vote choice. American Journal of Political Science, 58(1), 79-94. https://doi.org/10.1111/ajps.12046

Dimitrova, D. V., Shehata, A., Strömbäck, J. \& Nord, L. W. (2014). The effects of digital media on political knowledge and participation in election campaigns: Evidence from panel data. Communication Research, 41(1), 95-118. https://doi.org/10.1177/0093650211426004

Downs, A. (1957). An economic theory of democracy. Harper \& Row.

Farias Batlle, P. (Dir.) (2010). Informe anual de la profesión periodística 2010. Asociación de la Prensa de Madrid. https://www.apmadrid.es/publicaciones/informe-anual-de-la-profesion/

Fernández, C. B.y Rodríguez-Virgili,J. (2017). El consumo de información política de los públicos interesados comparado con el del electorado general: los casos de las elecciones de Argentina, España y Venezuela de 2015. Revista de Comunicación, 16(2), 60-87. https:// doi.org/10.26441/RC16.2-2017-A3 
Festinger, L. (1957). A theory of cognitive dissonance. Stanford University Press.

Fiorina, M. (1981). Retrospective voting in american national elections. Yale University Press.

Fletcher, R. \& Nielsen, R. K. (2017). Are news audiences increasingly fragmented? A cross-national comparative analysis of cross-platform news audience fragmentation and duplication. Journal of Communication, 67(4), 476-498. https://doi.org/10.1111/jcom.12315

Garrett, R. K. (2009). Echo chambers online? Politically motivated selective exposure among internet news users. Journal of Computer-Mediated Communication, 14(2), 265-285. https://doi.org/10.1111/ j.1083-6101.2009.01440.x

Garrett, R. K. (2013). Selective exposure: New methods and new directions. Communication Methods and Measures, 7(3-4), 247-256. https:// doi.org/10.1080/19312458.2013.835796

Gil de Zúñiga, H., Weeks, B. \& Ardèvol-Abreu, A. (2017). Effects of the news-finds-me perception in communication: Social media use implications for news seeking and learning about politics. Journal of Computer-Mediated Communication, 22(3), 105-123. https:// doi.org/10.1111/jcc4.12185

Graf, J. \& Aday, S. (2008). Selective attention to online political information. Journal of Broadcasting \& Electronic Media, 52(1), 86-100. https://doi.org/10.1080/08838150701820874

Hallin, D. C. \& Mancini, P. (2004). Comparing media systems: Three models of media and politics. Cambridge University Press.

Hernández-Santaolalla, V. (2018). Los efectos de los medios de comunicación de masas. UOC. 
Humanes Humanes, M. L. (2014). Exposición selectiva y partidismo de las audiencias en España: el consumo de información política durante las campañas electorales de 2008 y 2011. Palabra Clave, 17(3), 773-802. http://dx.doi.org/ 10.5294/pacla.2014.17.3.9

Iyengar, S. \& Hahn, K. S. (2009). Red media, blue media: Evidence of ideological selectivity in media use. Journal of Communication, 59(1), 19-39. https://doi.org/10.1111/j.1460-2466.2008.01402.x

Katz, E. \& Lazarsfeld, P. F. (1955). Personal influence: The part played by people in the flow of mass communications. Free Press.

Key, V. (1966). The responsible electorate: Rationality in presidential voting. Harvard University Press.

Kiewiet, D. R. \& Rivers, D. (1984). A retrospective on retrospective voting. Political Behavior, 6(4), 369-393. https://doi.org/10.1007/ BF00987073

Knobloch-Westerwick, S. (2012). Selective exposure and reinforcement of attitudes and partisanship before a presidential election. Journal of Communication, 62(4), 628-642. https://doi.org/10.1111/ j.1460-2466.2012.01651.x

Knobloch-Westerwick, S. (2015). Choice and preference in media use: Advances in selective exposure theory and research. Routledge.

Ksiazek, T. B. (2016). Partisan audience polarization: Beyond selective exposure. Atlantic Journal of Communication, 24(4), 216-227. https:// doi.org/10.1080/15456870.2016.1208658

Lazarsfeld, P., Berelson, B. \& Gaudet, H. (1944). The people's choice: How the voter makes up his mind in a presidential campaign. Duell, Sloan and Pearce. 
Lee, J. K. \& Kim, E. (2017). Incidental exposure to news: Predictors in the social media setting and effects on information gain online. Computers in Human Behavior, 75, 1008-1015. https://doi.org/10.1016/j. chb.2017.02.018

Lee, S. \& Xenos, M. (2019). Social distraction? Social media use and political knowledge in two US Presidential elections. Computers in Human Behavior, 90, 18-25. https://doi.org/10.1016/j.chb.2018.08.006

Levendusky, M. (2013). Partisan news that matters: How partisan news impact American politics. University of Chicago Press.

Lipset, S. M. \& Rokkan, S. (Eds.) (1967). Party systems and voter alignments: Cross-national perspectives. Free Press.

Martín-Llaguno, M. \& Berganza-Conde, M. R. (2001). Voters and mass media during 1996 spanish national election campaign: Selective exposure or media influence? Communication \& Society, 14(1), 51-70. https://revistas.unav.edu/index.php/communication-and-society/article/view/36382

Messing, S. \& Westwood, S. J. (2014). Selective exposure in the age of social media: Endorsements trump partisan source affiliation when selecting news online. Communication Research, 41 (8), 1042-1063. https://doi.org/10.1177/0093650212466406

Mitchell, A., Gottfried, J., Kiley, J. \& Matsa, K. E. (2014, octubre 21). Political polarization \& media habits. Pew Research Center. https://www. journalism.org/2014/10/21/political-polarization-media-habits/

Prior, M. (2013). Media and political polarization. Annual Review of Political Science, 16, 101-127. https://doi.org/10.1146/annurev-polisci-100711-135242

Rinke, E. \& Lück,J. (2010). Cognitive style, selectivity, and reinforcement: Toward clarifying the role of political ideology in the reception of 
political communication. En K. Mok \& M. Stahl, (Eds.), Politische kommunikation heute: Beiträge des 5. Düsseldorfer forums politische kommunication. (pp. 219-234). Frank \& Timme.

Rodriguez, C. G., Moskowitz, J. P., Salem, R. M. \& Ditto, P. H. (2017). Partisan selective exposure: The role of party, ideology and ideological extremity over time. Translational Issues in Psychological Science, 3(3), 254-271. https://doi.org/10.1037/tps0000121

Sears, D. O. \& Freedman, J. L. (1967). Selective exposure to information: A critical review. Public Opinion Quarterly, 31(2), 194-213. https:// doi.org/10.1086/267513

Shehata, A. \& Strömbäck, J. (2018). Learning political news from social media: Network media logic and current affairs news learning in a high-choice media environment. Communication Research.https:// doi.org/10.1177/0093650217749354

Stroud, N. J. (2008). Media use and political predispositions: Revisiting the concept of selective exposure. Political Behavior, 30(3), 341366. https://doi.org/10.1007/s11109-007-9050-9

Stroud, N.J. (2010). Polarization and partisan selective exposure. Journal of Communication, 60(3), 556-576. https://doi.org/10.1111/j.14602466.2010.01497.x

Tsfati, Y., Stroud, N. J. \& Chotiner, A. (2014). Exposure to ideological news and perceived opinion climate: Testing the media effects component of spiral-of-silence in a fragmented media landscape. The International Journal of Press/Politics, 19(1), 3-23. https://doi. org/10.1177/1940161213508206

Vraga, E. K. (2015). How party affiliation conditions the experience of dissonance and explains polarization and selective exposure. Social Science Quarterly, 96(2), 487-502. https://doi.org/10.1111/ ssqu. 12138 